J. Lake Sci.(湖泊科学) ,2008, 20(1): 76-82

http://www.jlakes.org. E-mail: jlakes@niglas.ac.cn

(C)2008 by Journal of Lake Sciences

\title{
太湖梅梁湾沉积岩芯元素地球化学记录的多元统计分析
}

\author{
林 琳 $^{1,2}$, 吴敬禄 ${ }^{1}$ \\ (1: 中国科学院南京地理与湖泊研究所湖泊与环境国家重点实验室, 南京 210008) \\ (2: 中国科学院研究生院, 北京 100049)
}

摘 要: 通过沉积岩芯元素含量垂直分布和富集因子分析, 结合多元统计方法如系统聚类和模糊聚类分析, 研究了太湖梅梁 湾沉积岩芯元素地球化学记录的湖泊环境演化过程. 以系统聚类分析和模糊聚类分析为基础, 将梅梁湾地球化学记录的环境 过程划分为如下几个层段: 0-6cm 为人类活动强烈干扰的阶段, $25-50 \mathrm{~cm}$ 和 $55-72 \mathrm{~cm}$ 层段都是自然过程的表现, 反映了流域物 源的影响，而两层段元素记录的差异反映了不同自然作用驱动下的湖泊环境变化; $6-25 \mathrm{~cm}$ 和 $50-55 \mathrm{~cm}$ 层段则是不同环境特征的 过渡阶段, 因此, 综合应用多元统计方法可以更直观更精确地量化影响湖泊环境的因素, 有助于恢复湖泊环境历史演化过程. 关键词: 梅梁湾; 太湖; 沉积物; 元素; 多元统计; 系统聚类

\section{Application of multivariate statistical analysis to elemental geochemical records of lacu- strine sediment of Meiliang Bay in Lake Taihu}

\author{
LIN Lin ${ }^{1,2} \&$ WU Jinglu ${ }^{1}$ \\ (1: State Key Laboratory of Lake Science and Environment, Nanjing Institute of Geography and Limnology, Chinese Academy of \\ Sciences, Nanjing 210008, P. R. China) \\ (2: Graduate School of the Chinese Academy of Sciences, Beijing 100049, P. R. China)
}

\begin{abstract}
Abstrast: To evaluate the environmental change of Meiliang Bay of Lake Taihu, the elemental geochemical records of the sediment core were analyzed by the element enrichment factors and multivariate statistical methods like hierarchical cluster and fuzzy cluster. The results of element enrichment factors showed the enrichment of heavy metals in surface layers. Hierarchical cluster analysis made it possible to separate several groups of elements with different sources or geochemical behaviors. Moreover, fussy C-means clustering method was applied to divide geochemical records of the sediment into several segments and separate natural factors from anthropogenic. The enrichment of heavy metals and nutrients above $6 \mathrm{~cm}$ depth was dominated by human activities. Both lower levels between 25 and $50 \mathrm{~cm}$ depth and higher levels between 55 and $72 \mathrm{~cm}$ depth of most elements had been driven by natural processes and been affected by catchment sources, and that discrimination between them indicated lacustrine environmental change driven by different natural processes. The transition periods on environment were reflected in other segments. Integrating these multivariate statistical methods is an efficient tool in achieving better understanding on the quantitative analysis for the complex factors that influence lakes, and helpful to reconstruct the history of environmental change in lakes.
\end{abstract}

Keywords: Meiliang Bay; Lake Taihu; sediments; elements; multivariate statistics; hierarchical clustering

湖泊沉积物可作为环境变化的信息档案库 ${ }^{[1]}$, 每一层沉积物都代表了与过去某一时段相关的环境条 件 ${ }^{[2]}$. 沉积物中的化学元素主要来源于流域汇水区域, 元素种类和含量取决于自然条件和过程(汇水区母 岩性质、流域气候条件和元素地球化学过程等)以及人类活动(工农业发展，人口及能源消耗的增长等)引 起的变化. 经定年沉积物垂直剖面的元素含量可用于流域自然环境演化和人为污染物排放来源的历史研 究 ${ }^{[3-4]}$. 由于沉积物记录了自然和人为因素的综合信息, 为研究人类活动对湖泊系统的影响, 需要沉积记录

* 国家自然科学基金项目(40673015)、中国科学院知识创新工程项目(KZCX2-YW-319)和科技部基础研究项目联合资助 (2002CB412300). 2006-12-13 收稿; 2007-04-14 收修改稿. 林琳, 女, 1979 年生, 博士研究生; E-mail: 1lin@niglas. ac. cn. 
剥离中反映的自然变化的信息进而识别人类活动的强度和方式, 这也是环境记录解释中的一个难点. 为 此，许多学者尝试通过学科交叉诊断污染来源和不同人类活动的响应. 近年来，统计学方法如相关分析、 主成分分析、聚类分析等在地学中的应用日渐深 ${ }^{[5-7]}$. 已有研究证明, 统计学方法可以削弱数据信息中 自然变化的影响并提高数据相互比较的统计能力, 从而为沉积记录的定量分析提供可能 ${ }^{[8-11]}$.

太湖流域是我国人口最密集、经济最发达的地区之一. 湖泊流域面积占国土面积 $0.38 \%$ ，人口占全国 的 $3.08 \%$. 太湖是上海、苏州、无锡、嘉兴等城市居民的主要供水水源 ${ }^{[12]}$. 近年来，由于工业排污、农业 面源污染、水产养殖污染及生活排污等影响，造成了湖泊富营养化、有机物和重金属污染等一系列环境 问题. 太湖人湖河道水质受污染程度呈明显上升趋势，河流中污染物含量持续增加，水质不断恶化，劣 于 $V$ 类的河流有 5 条，如无锡的梁溪河、直湖港，常州的武进港，苏州的月城河等 ${ }^{[13]}$. 太湖出人湖水量相 对于湖体容积较小, 而湖水滞留时间较长(309d), 如此增加和累积的污染物使得湖体水质污染呈上升趋 势, 特别是毗邻无锡市的五里湖和梅梁湾污染状况最为严重 ${ }^{[14]}$. 目前对太湖水污染问题已开展了广泛研 究 ${ }^{[15-18]}$, 但对沉积物元素地球化学特征及演化历史的分析还较少. 本文试图通过对太湖梅梁湾沉积岩芯 的元素含量分析并借助多元统计方法探讨该区元素地球化学的演化特征，从而揭示自然条件和人类活动 对湖体的影响.

\section{1 材料和分析方法}

\section{1 样品采集}

于 2004 年在梅梁湾(图 1)提取 $72 \mathrm{~cm}$ 柱状沉积 岩芯, 取芯过程中沉积物保持完好, 未受扰动. 现 场对岩芯表层 $20 \mathrm{~cm}$ 以 $0.5 \mathrm{~cm}$ 间隔, 其余以 $1 \mathrm{~cm}$ 间 隔切割, 密封保存后带回实验室. 室内将样品在 $40^{\circ} \mathrm{C}$ 烘干后用于粒度和元素含量分析.

\section{2 分析方法}

取少量样品 $(0.3 \mathrm{~g}$ 左右)加人过氧化氢和稀盐酸 去除有机质和碳酸盐, 洗至中性, 加人六偏磷酸钠 经超声波振荡分散后在 Malvern Mastersizer2000 型 激光粒度仪上进行粒度分析.

将样品研磨至 100 目后用于元素含量分析. 用 重铬酸钾-硫酸(油浴)氧化-硫酸亚铁滴定法测有机 碳(TOC)含量, 用重铬酸钾-硫酸消化-凯氏定氮法 测总氮 $(\mathrm{TN})$ 含量 ${ }^{[19]}$. 其它元素的测试是将样品加人

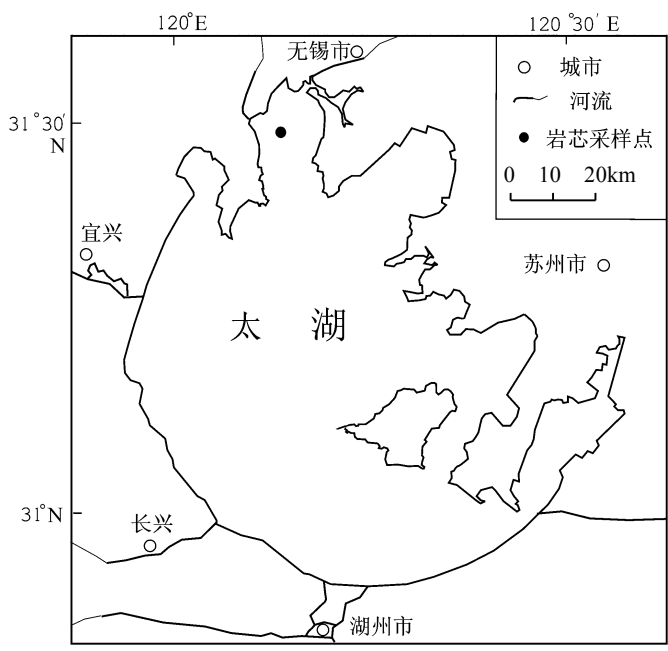

图 1 采样点位置

Fig. 1 Location of sampling site in Lake Taihu 硝酸、氢氟酸于 Berghof MWS-3 微波硝化系统中 硝化, 转移人聚四氟乙烯烧杯中加少量高氯酸蒸干, 用一定量稀硝酸和双氧水加热溶解残渣后, 用 Leeman Labs Profile ICP-AES 测定. 采用 SPEX CertiPreP ${ }^{\mathrm{TM}}$ Custom Assurance Standard 多元素标准溶液. 中国水系沉积物成份分析标准物质 GBW07311 作为标准参考物质.

\section{3 统计分析方法}

系统聚类分析和模糊C均值聚类分析分别用SPSS13.0 和Matlab6.5软件完成，聚类之前先将粒度和元 素数据用Z-score法标准化 ${ }^{[20]}$.

\section{2 结果}

\section{1 粒度和元素含量分析}

2.1 .1 剖面垂直分布 据该钻孔的年代学分析结果 ${ }^{[21]}$ 推断沉积岩芯 $0-12 \mathrm{~cm}$ 层段为 20 世纪 90 年代以来的沉 积, $30 \mathrm{~cm}$ 以下为 20 世纪 50 年代以前的沉积. 如图2所示岩芯的细粉砂 $(4-16 \mu \mathrm{m})$ 含量占优势, 平均 $47 \%$, 粘 土 $(<4 \mu \mathrm{m})$ 含量变化较稳定, 平均 $18 \%$ 左右, 粉砂 $(16-64 \mu \mathrm{m})$ 含量波动范围较大, 平均约占 $34 \%$. 除个别元 
素以外，大部分元素含量在深度 $72-55 \mathrm{~cm}$ 段是高值区， $55 \mathrm{~cm}$ 以上明显下降，在 $55-30 \mathrm{~cm}$ 为低值区， $30 \mathrm{~cm}$ 开 始有上升的趋势, $12 \mathrm{~cm}$ 以上元素间有明显差异，其中重金属和营养元素含量迅速增长, $\mathrm{Al} 、 \mathrm{Fe} 、 \mathrm{Co}$ 则保持 稳定.

2.1.2 富集因子分析 为了量化金属元素在沉积剖面上的分布从而分析其富集或损耗的原因，可以用富 集因子来初步分析 ${ }^{[22]}$. 富集因子就是将新沉积 $(0-1 \mathrm{~cm}$ 深度)的元素含量和本底元素含量的比值. 比值大 于1表示元素富集，小于1则表示元素损耗，沉积物元素本底值的确定通常是将沉积岩芯定年后选取认为 未受人类活动干扰层段元素含量的平均值 ${ }^{[23]}$. 据以前研究结果太湖沉积物受下蜀黄土的影响较大, 选下 蜀黄土为本底更客观 ${ }^{[1,24]}$. 以不同年代各层段元素含量平均值与以下蜀黄土元素含量 ${ }^{[25]}$ 为本底计算富集 因子(表1)，结果表明表层和30cm以下层段(1950年以前) $\mathrm{Ni} 、 \mathrm{~Pb} 、 \mathrm{Zn}$ 等重金属元素明显富集.

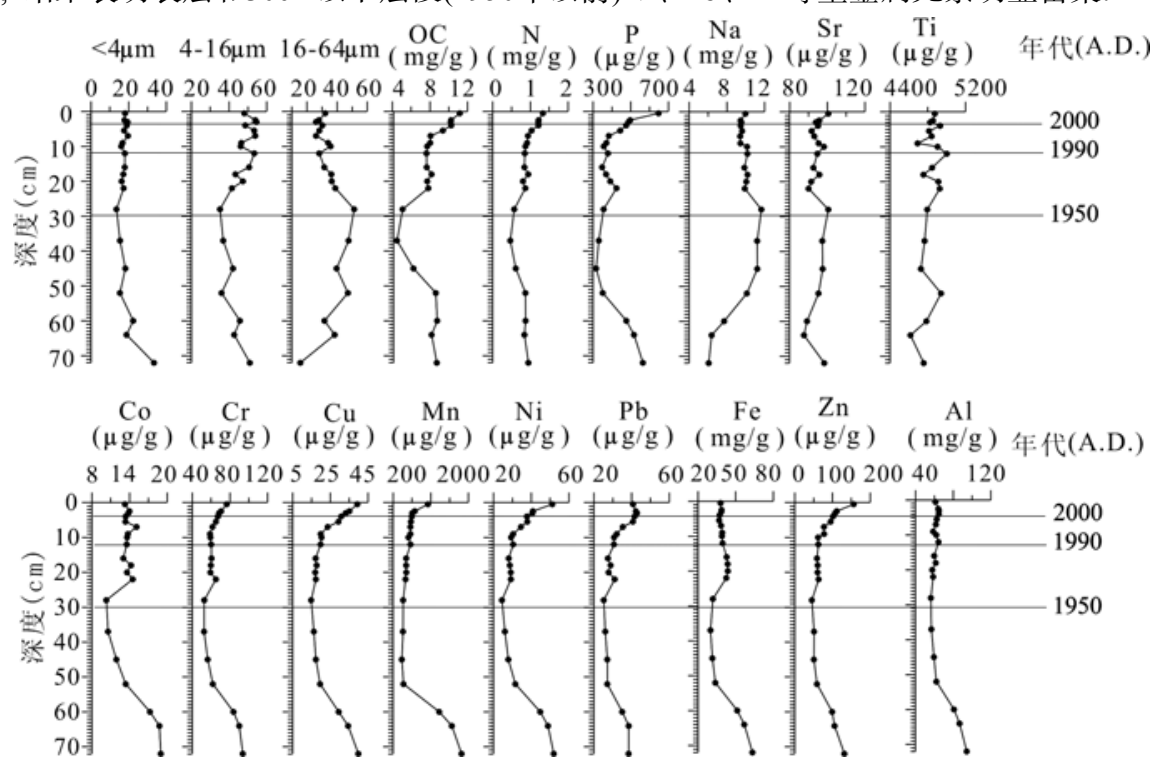

图2 沉积岩芯粒度及元素含量随深度变化

Fig. 2 The distribution with depth of grain size and element concentrations of the sediment core

表1 金属元素含量 $(\mu \mathrm{g} / \mathrm{g})$ 及富集因子

Tab. 1 The concentrations $(\mu \mathrm{g} / \mathrm{g})$ and enrichment factors of the metals

\begin{tabular}{ccccccccc}
\hline 深度(cm) & 年代(A. D. ) & $\mathrm{Co}$ & $\mathrm{Cr}$ & $\mathrm{Cu}$ & $\mathrm{Mn}$ & $\mathrm{Ni}$ & $\mathrm{Pb}$ & $\mathrm{Zn}$ \\
\hline $0-1$ & 2004 & 13.3 & 76 & 39.3 & 1032 & 51.2 & 41 & 156 \\
$0-12$ & $1990-2004$ & 13.8 & 65.3 & 27.9 & 675 & 37 & 37 & 95 \\
$12-30$ & $1950-1990$ & 13.1 & 59.4 & 16.8 & 493.7 & 27.9 & 28.0 & 57.6 \\
$30-72$ & 1950 前 & 15.1 & 72.9 & 26.1 & 1003.7 & 38.5 & 32.0 & 82.7 \\
下蜀黄土 & & 15.6 & 69.4 & 31.4 & 625 & 29.9 & 15.7 & 66 \\
富集因子 $^{(1}$ & & 0.85 & 1.10 & 1.25 & 1.65 & 1.71 & 2.59 & 2.37 \\
富集因子 $^{(2)}$ & & 0.88 & 0.94 & 0.89 & 1.08 & 1.24 & 2.38 & 1.43 \\
富集因子 $^{(3)}$ & & 0.84 & 0.86 & 0.53 & 0.79 & 0.93 & 1.79 & 0.87 \\
富集因子 $^{(4}$ & & 0.97 & 1.05 & 0.83 & 1.61 & 1.29 & 2.04 & 1.25 \\
\hline
\end{tabular}

(1)、(2)、(3)、(4)分别表示 $0-1 \mathrm{~cm} 、 0-12 \mathrm{~cm} 、 12-30 \mathrm{~cm}$ 和 $30-72 \mathrm{~cm}$ 层段元素富集因子. 


\section{2 统计分析结果}

2.2.1 系统聚类分析 聚类分析是根据指标间的相关性或者是样品间的相似性对指标或样品归类的一种多 元分析方法, 当确定了样品或变量间的距离或相似系数后, 就要对样品或变量进行分类, 分类的方法很 多，一类方法是在样品距离的基础上定义类与类之间的距离，首先将 $\mathrm{m}$ 个样品自成一类，然后每次将具有最 小距离的两类合并, 合并后重新计算类与类之间的距离, 这个过程一直继续到所有样品归为一类为止. 把 这个过程作成一张聚类谱系图(Hierarchical diagram), 这种聚类方法称为系统聚类法 (又称谱系聚类) ${ }^{[26]}$. 通过系统聚类法对岩芯粒度和元素指标进行聚类(图 3), 图中横坐标代表了不同指标间的距离, 距离越近, 则指标间相似性越高. $\mathrm{OC}$ 和 $\mathrm{N}, \mathrm{Cu} 、 \mathrm{Zn} 、 \mathrm{Ni}$ 和 $\mathrm{P}, \mathrm{Cr} 、 \mathrm{Mn}$ 和 $\mathrm{Al}, \mathrm{Co}$ 和 $\mathrm{Fe}$ 间的距离都小于 2, 说明他们具 有相同的来源或者相似的地球化学行为. $\mathrm{OC} 、 \mathrm{~N} 、 \mathrm{P}$ 和重金属 $\mathrm{Cu} 、 \mathrm{Zn} 、 \mathrm{Ni} 、 \mathrm{~Pb}$ 与细粉砂为一组, 可以反 映人类活动的影响. $\mathrm{Cr} 、 \mathrm{Mn} 、 \mathrm{Al} 、 \mathrm{Co}$ 和 $\mathrm{Fe}$ 与粘土含量相关, 说明这些元素主要受沉积环境的制约. 但上 述元素与易迁移的 $\mathrm{Na} 、 \mathrm{Sr}$ 以及难迁移的 $\mathrm{Ti}$ 距离较远，反映了不同类间元素的组合特征.

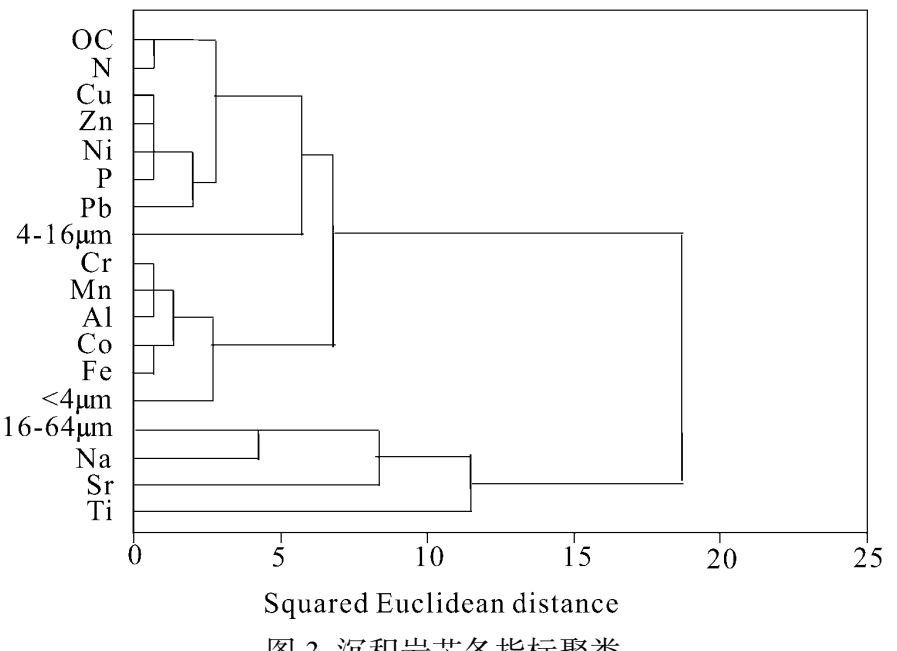

Fig. 3 Hierarchical diagram of indexes of the sediment core

2.2.2 模糊聚类分析一般聚类方法是把样本按照某种算法归为某一类, 但在科学研究中存在着许多定义 不很严格或者说具有模糊性概念的事物，这就造成不同类间样本缺少相互联系. 这里所谓的模糊性，主 要是指客观事物的差异在中间过渡中的不分明性 ${ }^{[27]}$. 因此把模糊数学方法引人聚类分析, 就能使分类更 切合实际. 模糊聚类法是利用模糊集理论来处理分类问题, 它对研究领域中具有模糊特征的两态数据或 多态数据具有明显的分类效果，根据一定的隶属度来确定其分类关系 ${ }^{[28]}$. 根据系统聚类的分析结果，选 取 $\mathrm{OC} 、 \mathrm{Zn} 、 \mathrm{~Pb} 、 \mathrm{Al} 、 \mathrm{Fe} 、 \mathrm{Sr} 、 \mathrm{Ti}$ 作为典型指标，对沉积岩芯进行模糊 $\mathrm{C}$ 均值聚类，把样品依次划分为 2 、 $3 、 4$ 类(图 4、图 5). 当样品聚为 2 类时，表层 6-0 cm 样品属于第一类，其隶属度在 $70 \%-91 \%$ 之间, $55-6 \mathrm{~cm}$ 样品属于第二类的可能性在 $60 \%$ 以上，属于第一类的可能性在 $36 \%$ 以下，则这些样品属于第二类， $72-55 \mathrm{~cm}$ 样品属于第一类的隶属度平均为 $65 \%$ (图 $4 \mathrm{~A}$ ). 而 $6-0 \mathrm{~cm}$ 和 $72-55 \mathrm{~cm}$ 为 $\mathrm{Pb} 、 \mathrm{Zn}$ 和有机碳高值区 (图 5A). 当样品聚为三类时，原第二类中 $50-25 \mathrm{~cm}$ 样品被划为第三类，其隶属度在 $84 \%-94 \%$ 之间，这些 样品 $\mathrm{OC} 、 \mathrm{Zn}$ 和 $\mathrm{Pb}$ 含量较低而 $\mathrm{Sr}$ 含量较高, $72-55 \mathrm{~cm}$ 样品属于第一类的可能性在 $44 \%$ 左右, 属于第三类 的可能性占 35\%左右(图 4B, 图 5B). 聚为 4 类时, 原第一类的 $72-55 \mathrm{~cm}$ 样品被划为第四类(图 4C), 其隶 属度在 55\%-83\%之间, 聚类特征图(图 5C) 上样品 $\mathrm{Sr} 、 \mathrm{Ti}$ 含量低其它元素含量高. 当再尝试分更多的类时, 各类特征已不明显.

\section{3 讨论与结论}

梅梁湾沉积岩芯的粒度特征和元素含量曲线(图 2)与刘恩峰等 ${ }^{[29]}$ 在太湖马迹山附近湖区沉积岩芯及 
刘建军等 ${ }^{[30]}$ 在大浦湖区沉积岩芯的结果不一致, 可能与不同湖区物质来源及水体的沉积过程有关. 因 此, 太湖不同湖区沉积岩芯元素地球化学记录了区域的环境特征，反映了区域自然环境及人类活动的影 响过程.

通过富集因子分析发现表层和 1950 年以前沉积物重金属元素明显富集. 根据太湖 1950 年以来水环 境监测资料, 20 世纪 50-60 年代水环境质量良好, 受人类活动影响不明显 ${ }^{[31]}$. 而且从图 2 看, 1950 年以前 沉积物剖面 $\mathrm{Fe} 、 \mathrm{Al}$ 元素和重金属元素含量变化一致. 由于沉积物中 $\mathrm{Fe} 、 \mathrm{Al}$ 元素含量不受污染物输人影

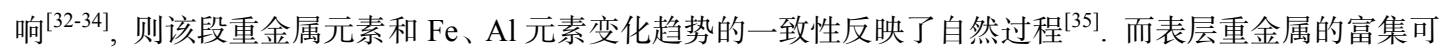
能与工业污染物排放有关, 反映了工业活动的影响.

应用系统聚类分析将沉积岩芯的粒度和元素指标分成具有不同特征的元素组合, 包括反映人类活动 的营养元素和重金属; 受沉积环境制约的 $\mathrm{Cr} 、 \mathrm{Mn} 、 \mathrm{Al} 、 \mathrm{Co}$ 和 $\mathrm{Fe}$; 还有易迁移的元素 $\mathrm{Na} 、 \mathrm{Sr}$ 和难迁移的 Ti. 系统聚类简化了分析过程, 有助于在每一类中选择相应的元素来详细分析岩芯垂直分布特征, 但不 能反映影响沉积岩芯不同层段元素特征的多种环境因子.

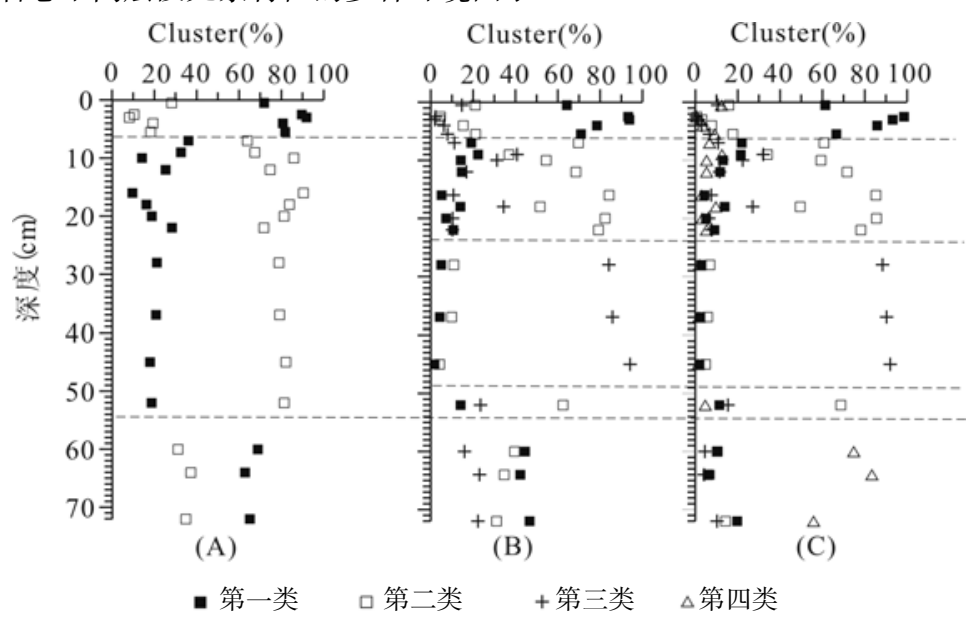

图 4 沉积岩芯模糊 $\mathrm{C}$ 均值聚类分布 $(\mathrm{A} 、 \mathrm{~B}$ 和 $\mathrm{C}$ 分别是聚类 $2 、 3 、 4$ 类时的分布)

Fig. 4 Results of fussy c-means clustering of the sediment core

(A, B and C indicate the distribution of two, three and four clusters)

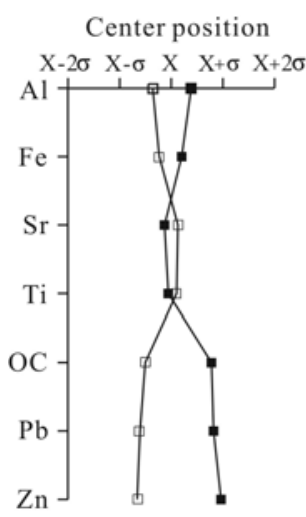

(A)

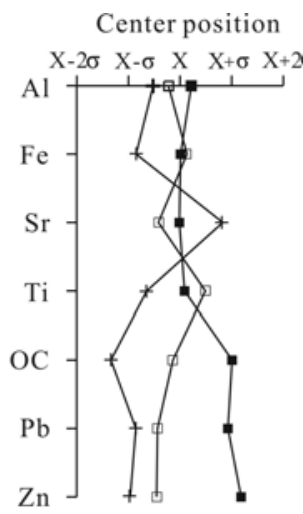

(B)

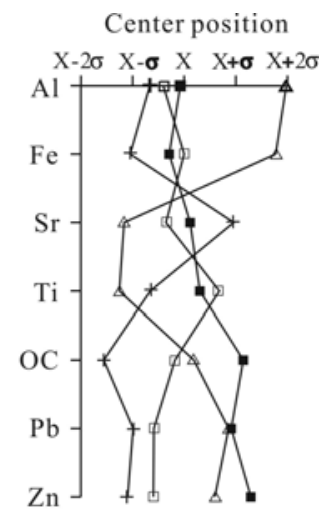

(C)

- 第一类 口第二类 +第三类 $\triangle$ 第四类

图 5 沉积岩芯模糊 $\mathrm{C}$ 均值聚类特征图( $\mathrm{A} 、 \mathrm{~B}$ 和 $\mathrm{C}$ 分别是聚类 $2 、 3 、 4$ 类时的特征图)

Fig. 5 Characteristics of fussy c-means clustering of the sediment core

(A, B and $\mathrm{C}$ indicate the characteristics of two, three and four clusters) 
模糊聚类方法是以样本归为某类的隶属度来分类，每个类间没有严格的界限，用户可以尝试划分不 同的类, 并结合实际寻找合理的聚类结果 ${ }^{[36]}$. 从模糊聚类的过程和元素分析可以看出, $6-0 \mathrm{~cm}$ 为营养元 素和重金属元素大量富集的层段，说明工农业和生活污水的影响. 50-25cm 层段 $\mathrm{Pb} 、 \mathrm{Zn}$ 含量较低反映人 类活动的影响较小, 而且 $\mathrm{Sr}$ 是易迁移的元素，在沉积物中 $\mathrm{Sr}$ 含量增长反映了源区物质输人的过程，这说 明该段沉积环境主要体现了流域物源的影响 ${ }^{[37-38]}$. $72-55 \mathrm{~cm}$ 层段, 不同于其它层段, 该段 $\mathrm{Sr} 、 \mathrm{Ti}$ 含量较 低, $\mathrm{Al} 、 \mathrm{Fe}$ 含量较高, 可能反映了沉积环境或流域物质来源的不一致. 根据其他研究者对太湖沉积物的研 究, 对应层段碳、氮元素含量等地球化学指标也显示为含量高值区, 可能反映湖泊流域经历较大的环境 变迁, 造成人湖物质来源的变化 ${ }^{[39]}$. 本文岩芯底部元素含量高值段与其他研究的比较结果反映了太湖不 同湖区早期沉积具有较好的一致性.

致谢: 中国科学院南京地理与湖泊研究所王苏民研究员在论文写作过程中提出了有益的建议, 朱育新副 研究员和隋桂荣老师帮助完成元素和有机碳、氮含量分析. 作者在此表示衰心的感谢.

\section{4 参考文献}

[1] Haworth EY, Lund JWG eds. Lake Sediments and Environmental History. Minneapolis: University of Minnesota Press, 1984: 411.

[2] Von Gunten HR, Sturm M, Moser RN. 200-year record of metals in lake sediments and natural background concentrations. Environ Sci Technol, 1997, 31(8): 2193-2197.

[3] Palanques A, Sanchez Cabeza JA, Masqué P et al. Historical record of heavy metals in a highly contaminated Mediterranean deposit: The Besòs prodelta. Mar Chem, 1998, 61(3-4): 209-217.

[41 Birch L, Hanselmann KW, Bachofen R. Heavy metal conservation in Lake Cadagno sediments: historical records of anthropogenic emissions in a meromictic alpine lake. Wat Res, 1996, 30(3): 679-687.

[5] Nguyen HL, Leermakers M, Elskens $\mathrm{M}$ et al. Correlations, partitioning and bioaccumulation of heavy metals between different compartments of Lake Balaton. Sci Total Environ, 2005, 341: 211-226.

[6] Samecka-Cymerman A, Kempers AJ. Concentrations of heavy metals and plant nutrients in water, sediments and aquatic macrophytes of anthropogenic lakes (former open cut brown coal mines) differing in stage of acidification. Sci Total Environ, 2001, 281(1-3): 87-98

[7] Arnason JG, Fletcher BA. A $40^{+}$year record of $\mathrm{Cd}, \mathrm{Hg}, \mathrm{Pb}$, and $\mathrm{U}$ deposition in sediments of Patroon Reservoir, Albany County, NY, USA. Environ Pollut, 2003, 123(3): 383-391.

[8] Einax JW, Soldt U. Geostatistical and multivariate statistical methods for the assessment of polluted soils-merits and limitations. Chemometr Intell Lab, 1999, 46: 79-91.

[9] Simeonov V, Andreev G. Interpretation of Black Sea sediments analytical data by the clustering approach. Toxicol Environ Chem, 1989, 24: 233-240.

[10] Hanson PJ, Evans DW, Colby DR et al. Assessment of elemental contamination in estuarine and coastal environments based on geochemical and statistical modeling of sediments. Mar Environ Res, 1993, 36(4): 237-266.

[11] Simeonov V, Massart DL, Andreev G et al. Assessment of metal pollution based on multivariate statistical modeling of 'hot spot' sediments from the Black Sea. Chemosphere, 2000, 41: 1411-1417.

[12] 杨桂山, 王德建. 太湖流域经济发展、水环境、水灾害. 北京: 科学出版社, 2003: 3-89.

[13] 王苏民, 穼鸿身. 中国湖泊志. 北京: 科学出版社, 1998: 261-268.

[14] 孙顺才, 黄渏平. 太湖. 北京: 海洋出版社, 1993: 1-234.

[15] Rose NL, Boyle JF, Du Y et al. Sedimentary evidence for changes in the pollution status of Taihu in the Jiangsu region of eastern China. J Paleolimn, 2004, 32(1): 41-51.

[16] 袁旭音, 王 禹, 孙 成等. 太湖底泥中多氯联苯的特征与环境效应. 长江流域资源与环境, 2004, 13(3): 272-276.

[17] 姚书春, 李世杰, 薛 滨等. 南太湖沉积岩芯中金属和营养元素的垂向分布特征及其意义. 生态环境, 2005, 14(2): 178-181.

[18] 范成新, 朱育新, 龚志军等. 太湖宜溧河水系沉积物的重金属污染特征. 湖泊科学, 2002, 14(3): 235-241. 
[19] 金相灿，屠清瑛主编. 湖泊富营养化调查规范(第二版). 北京：中国环境科学出版社, 1990: 159-206.

[20] 张文璋主编. 实用统计分析方法与 SPSS 应用. 北京: 机械工业出版社, 2000: 221-240.

[21] 林 琳, 吴敬禄. 太湖梅梁湾富营养化过程的同位素地球化学证据. 中国科学(D 辑), 2005, 35(增刊 II ): 55-62.

[22] Lockhart WL. Implications of chemical contaminants for aquatic animals in the Canadian Arctic: some review comments. Sci Total Environ, 1995, 160/161: 631-641.

[23] Rollinson HR ed. Using Geochemical Data: Evaluation, Presentations, Interpretation. New York: John Wiley \& Sons, 1992: $1-343$.

[24] 张于平, 翟文川. 太湖沉积物中重金属的测定及环境意义. 岩矿测试, 2001, 20(1): 34-36.

[25] 环境科学编辑部主编. 环境中若干元素的自然背景值及其研究方法. 北京: 科学出版社, 1982: 16-26.

[26] 陈平雁, 黄浙明主编. SPSS10.0 统计软件应用教程. 北京: 人民军医出版社, 2002: 165-175.

[27] 汪培庄主编. 模糊集合论及其应用. 上海: 上海科学技术出版社, 1983: 1-123.

[28] Stallings W ed. Cryptography and Network Security: Principles and Practice. New Jersey: Prentice Hall, 1999 : 1-569.

[29] 刘恩峰, 沈 吉, 刘兴起等. 太湖沉积物重金属和营养盐变化特征及污染历史. 中国科学(D 辑), 2005, 35(增刊 II): 73-80.

[30] 刘建军, 吴敬禄. 太湖大浦湖区近百年来湖泊记录的环境信息. 古地理学报, 2006, 8 (4): 559-564.

[31] 秦伯强, 胡维平, 陈伟民等. 太湖水环境演化过程与机理. 北京: 科学出版社, 2004: 19-33.

[32] Forstner U, Wittmann GTW eds. Metal pollution in the aquatic environment. New York: Springer, 1981.

[33] Loring DH. Lithium-a new approach for the granulometric normalization of trace metal data. Mar Chem, 1990, 29: 155-168.

[34] Luoma SN. Processes affecting metal concentrations in estuarine and coastal marine sediments. In: Furnes RW, Rainbow PS eds. Heavy metals in the marine environment. Boca Raton: CRC Press, 1990.

[35] Chih-An Huh. Fluxes and budgets of anthropogenic metals in the Santa Monica and San Pedro Basins off Los Angeles-review and reassessment. Sci Total Environ, 1996, 179(1-3): 47-60.

[36] 陈桂明, 戚红雨, 潘 伟主编. Matlab 数理统计(6.x). 北京: 科学出版社, 2002: 189 .

[37] 金章东, 王苏民, 沈 吉等. 小冰期弱化学风化的湖泊沉积记录. 中国科学(D 辑), 2001, 31(3): 221-225.

[38] 熊尚发, 丁仲礼, 刘东生. 南方红土元素迁移特征及其古环境意义. 土壤学报, 2001, 38(1): 25-31.

[39] 薛 滨, 㫿文川, 吴艳宏等. 太湖冕冰期-全新世气候环境变化的沉积记录. 湖泊科学, 1998, 10(2): 30-36. 